\title{
Modular Cyclopentenone Synthesis through the Catalytic Molecular Shuffling of Unsaturated Acid Chlorides and Alkynes
}

\section{Journal Article}

Author(s):

Lee, Yong Ho (1); Denton, Elliott (1); Morandi, Bill

Publication date:

2020-12-16

Permanent link:

https://doi.org/10.3929/ethz-b-000463053

Rights / license:

In Copyright - Non-Commercial Use Permitted

Originally published in:

Journal of the American Chemical Society 142(50), https://doi.org/10.1021/jacs.0c10832

Funding acknowledgement:

757608 - Shuttle Catalysis for Reversible Molecular Construction (EC) 
This document is the Accepted Manuscript version of a Published Work that appeared in final form in the Journal of the American Chemical Society, copyright (C) American Chemical Society after peer review and technical editing by the publisher. To access the final edited and published work see https://doi.org/10.1021/jacs.0c10832

\title{
Modular cyclopentenone synthesis through the catalytic molecular shuffling of unsaturated acid chlorides and alkynes
}

\author{
Yong Ho Lee, Elliott H. Denton, Bill Morandi* \\ ETH Zürich, Vladimir-Prelog-Weg 3, HCI, 8093 Zürich, Switzerland
}

\begin{abstract}
We describe a general strategy for the intermolecular synthesis of polysubstituted cyclopentenones using palladium catalysis. Overall, this reaction is achieved via a molecular shuffling process involving an alkyne, an $\alpha, \beta$-unsaturated acid chloride, which serves as both the alkene and carbon monoxide source, and a hydrosilane to create three new $\mathrm{C}-\mathrm{C}$ bonds. This new carbon monoxide-free pathway delivers the products with excellent yields. Furthermore, the regioselectivity is complementary to conventional methods for cyclopentenone synthesis. In addition, a set of regio- and chemodivergent reactions are presented to emphasize the synthetic potential of this novel strategy.
\end{abstract}

The construction of multiple $\mathrm{C}-\mathrm{C}$ bonds in a single reaction allows for a dramatic increase in molecular complexity. ${ }^{1,2}$ These reactions, when modular, allow for rapidly generating of libraries of complex molecules. ${ }^{3}$ As part of our investigation into palladium-catalyzed carbofunctionalizations, we recently reported such a modular process best described as an intermolecular carboformylation of alkynes. ${ }^{4}$ A molecular shuffling strategy was key to orchestrate a sequential bond formation in a programmed order: (1) an aromatic acid chloride was first deconstructed to its aryl, $\mathrm{CO}$, and $\mathrm{Cl}$ fragments by a Pd catalyst; (2) the individual fragments on the Pd center were then merged via consecutive carbometallation of an alkyne, $\mathrm{CO}$ reinsertion and $\mathrm{C}-\mathrm{H}$ reductive elimination, to provide the carboformylated compounds with excellent stereoselectivity.

We thus questioned whether this unique reactivity pattern could be further utilized in the construction of cyclopentenones. ${ }^{5,6}$ These highly functionalized motifs, commonly found in natural products, bioactive compounds, and key synthetic building blocks, are often accessed intramolecularly via the Pauson-Khand reaction $(\mathrm{PKR})^{7,8}$ or Nazarov cyclization. ${ }^{9,10}$ This strategy can be useful but often results in an inherent lack of flexibility given the need for the synthesis of the intermediate over several steps, undermining its potential. By contrast, the intermolecular PKR enables chemists to envision a simple 1 step synthesis of the cyclopentenones, yet the feasibility is traditionally limited (Scheme 1a, left). ${ }^{11,12}$ Specific challenges are (1) alkenes show low reactivity; (2) unreliable control of regioselectivity with respect to the alkene component, and (3) the requirement of excess hazardous chemicals (e.g. pressurized CO gas or stoichiometric cobalt-carbonyl complexes). While several creative solutions have been demonstrated to overcome specific challenges, such as the alkene scope being improved by the pre-installation of a cleavable directing group, ${ }^{13,14}$ the use of CO surrogates ${ }^{15}$ and less toxic metal catalysts/reagents, ${ }^{16,17}$ these developments have not yet provided a general solution. Instead, approaches that are mechanistically distinct from the PKR, introduce paradigms that are often complementary in nature. One such example is the development of cyclization reactions of unsaturated carbonyl compounds and alkynes to generate cyclopentenones using $\mathrm{Co} / \mathrm{Rh}$ and $\mathrm{Ni}$ catalysts, respectively (Scheme 1a, right)..$^{18,19}$

Scheme 1. Context of the work 

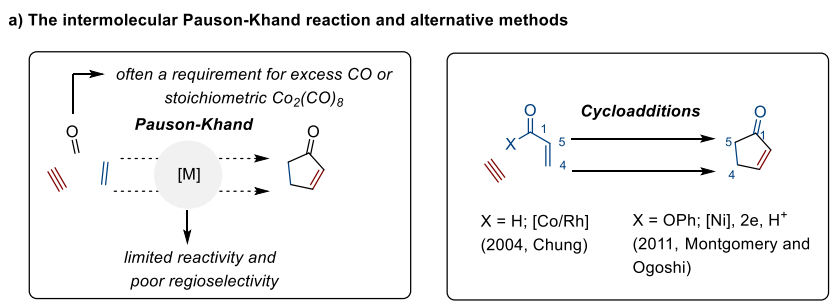

b) Our hypothesis

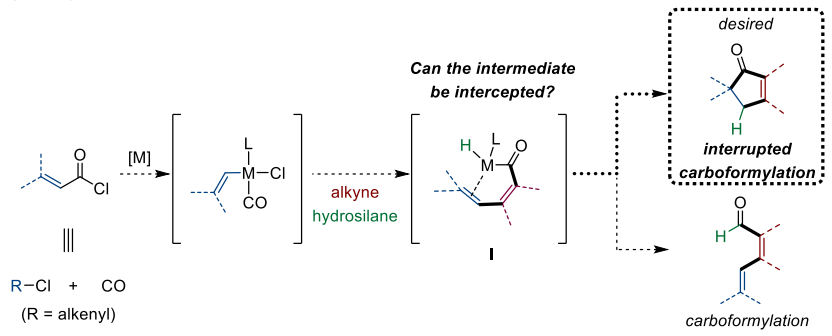

c) This work: interrupted carboformylation to deliver substituted cyclopentenones

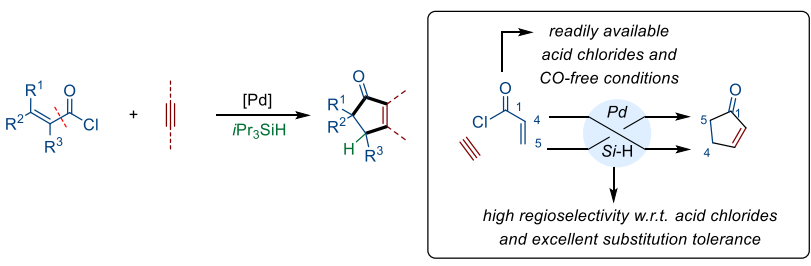

Table 1. Reaction Optimization

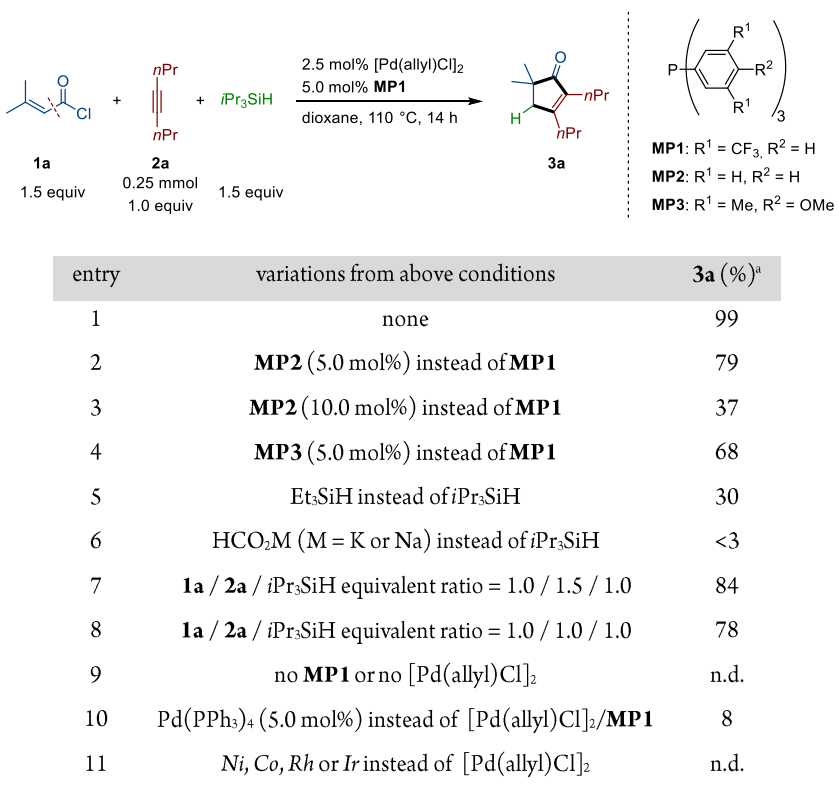

aThe GC yields are based on the moles of a limiting reagent versus $n$ dodecane, an internal standard.
We hypothesized that we could utilize the previously described carboformylation process as a distinct manifold for the intermolecular creation of carbocycles with a unique substitution pattern (Scheme 1b) ${ }^{20,21}$ Specifically, we questioned if a proposed key intermediate of the carboformylation reaction, $\mathbf{I}$, could be diverted to undergo an intramolecular cyclization. Such a framework for cyclopentenone synthesis could, in principle, offer several complementary features when compared to the state-of-the-art: (1) an alternative de novo reaction design where a wide range of alkene substrates can be tolerated; (2) safe and practical application due to the absence of pressurized $\mathrm{CO}$ as a reagent; (3) facile creation of a quaternary carbon center at the 5-position, a rare substitution pattern. Herein, we report the successful realization of an intermolecular PK-type reaction of internal alkynes using an $\alpha, \beta$-unsaturated acid chloride as an alkenyl and CO source (Scheme 1c).

As a model reaction for our initial investigations, an $\alpha, \beta$-unsaturated acid chloride (1a) and an alkyne (2a) were combined with a $\mathrm{Pd}$ precatalyst and a range of phosphine ligands that have been shown to mediate the reversible decarbonylation of acid chlorides. ${ }^{4,22}$ Gratifyingly, we identified several suitable phosphine ligands for this reaction. Importantly, the carboformylation product was not observed to any detectable extent, effectively validating our hypothesis that the carboformylation process can indeed be interrupted to deliver cyclopentenones (see SI). A systematic investigation led to the best yields of $\mathbf{3 a}$ being obtained with an electron deficient monophosphine ligand, MP1 (Table 1 and see SI). While triarylphosphines other than MP1 resulted in moderated yields of 3a, the results show a dependence on both the ratio of $\mathrm{P}$ to $\mathrm{Pd}$ and the electronics of the phosphine ligands (Table 1 and see SI). An increased $\mathrm{P}$ to $\mathrm{Pd}$ ratio, particularly with more basic or sterically less hindered phosphines, impeded the reaction. Presumably, this decreased reactivity is due to hampered alkenyl coordination to the $\mathrm{Pd}$ species, which is required for the intramolecular cyclization. ${ }^{23}$

The yield of the desired product increased proportionally to the steric bulk of the trialkylsilane (Table 1 , entry 5 and see SI). An inexpensive bulky silane, triisopropylsilane $\left(i \mathrm{Pr}_{3} \mathrm{SiH}\right)$, proved optimal. Alternative hydride sources, such as formate salts, failed to deliver the product (Table 1, entry 6). We performed an experiment using $\mathbf{1 a}$ as a limiting reagent, as well as a separate experiment using equimolar amounts of all reagents, to demonstrate the reaction's efficiency. Both reactions afforded $3 \mathbf{a}$ in good yields of $84 \%$ and $78 \%$, respectively (Table 1, entry 7 and 8 ). This result highlights the high efficiency with respect to the $\mathrm{CO}$ surrogate. ${ }^{24}$ The catalyst system was found to be highly active, with lower catalyst loadings of 1.0, 0.5 and $0.2 \mathrm{~mol} \%$ giving the desired product in 95,82 and $53 \%$ yield respectively (see SI). In the absence of either the ligand or Pd precatalyst, the formation of $\mathbf{3 a}$ was not observed (Table 1, entry 9). Other transition metal catalysts such as $\mathrm{Ni}(\operatorname{cod})_{2}, \mathrm{Co}_{2}(\mathrm{CO})_{8}$, $[\mathrm{Rh}(\operatorname{cod}) \mathrm{Cl}]_{2}$ or $[\mathrm{Ir}(\operatorname{cod}) \mathrm{Cl}]_{2}$ did not afford 3a (Table 1 , entry 11 and see SI for details).

We next explored the substrate scope of this transformation (Table 2). $\beta$-Dialkyl, $\beta$-diarylsubstituted and $\beta$-monosubstituted enoyl chlorides are well tolerated, giving a single regioisomer in each instance. We were able to confirm the structure of compound $3 \mathbf{e}$ by $\mathrm{X}$ ray crystallography. Notably, we could construct a variety of challenging quaternary carbon centers demonstrated by several novel ex- 
amples (3b-3f), including spirocycles. Previously, this rare substitution pattern has only ever been formed in Pauson-Khand cycloaddition reactions as a minor regioisomer with highly strained alkenes. $^{25,26}$ Sterically hindered $\beta$-aryl enoyl chlorides (3k-3o) provided excellent yields while other aryl-substituted acid chlorides resulted in moderate to good yields, regardless of electronic properties. A large variety of functional groups including ethers $(\mathbf{3 b}, \mathbf{3 1}, \mathbf{3 q}$, 3r, 3ac), alkenes (3d), carbamates (3f), nitriles (3z, 3an), esters (3m, 3w), aldehydes (3x), sulfones (3y), halogens (F: 3u, 3ad, 3af, $\mathrm{Cl}: \mathbf{3 o}, \mathbf{3 s}, \mathrm{Br}$ : $\mathbf{3 t}$ and even I: $\mathbf{3 a b}$ ) and nitro groups (3aa) were compatible under the reaction conditions. It is noteworthy that the reductively labile groups (e.g. esters, halides etc.) survived the reductive reaction conditions. Acid chlorides bearing heterocycles such as thiophenes (3ah, 3aj) and furans (3ag, 3ai) are also effective reaction partners.

Conveniently, the reaction can be performed directly from the $\alpha, \beta$-unsaturated carboxylic acid, when combined with Ghosez's reagent, for in situ formation of the acid chloride $(\mathbf{3 d}, \mathbf{3 f}, \mathbf{3 x}) \cdot{ }^{27}$ In this Table 2. Scope with respect to a, $\beta$-unsaturated acid chlorides ${ }^{a}$ manner, geranic acid was smoothly converted to the corresponding cyclopentenone (3d).

Remarkably, acryloyl chloride could be used both as an ethene and $\mathrm{CO}$ surrogate (3ak), addressing a classical limitation of the conventional PKRs. ${ }^{28}$ Further, the reaction also works with both $\alpha$-substituted and $\alpha, \beta$-disubstituted enoyl chlorides bearing methyl (3al), fluoro (3am), ${ }^{29}$ cyano (3an) and phenyl (3ao) groups, to furnish a single diastereomer in these cases bearing an additional $\beta$-substituent in moderate to low yield. In the reaction of 2,3-diphenylacryloyl chloride, an additional product (3ao-2) arising from isomerization of the desired product, was also observed. Likewise, the reaction with sorbic acid chloride bearing a conjugated diene functionality also resulted in isomerization of the distant alkene to give $3 \mathrm{ar}(E / Z$ $=82: 18$, separable $E / Z$ isomers $).{ }^{17 \mathrm{~d}}$ To further test variation in the substitution pattern of the $\alpha, \beta$-unsaturated acid chloride, fully substituted 


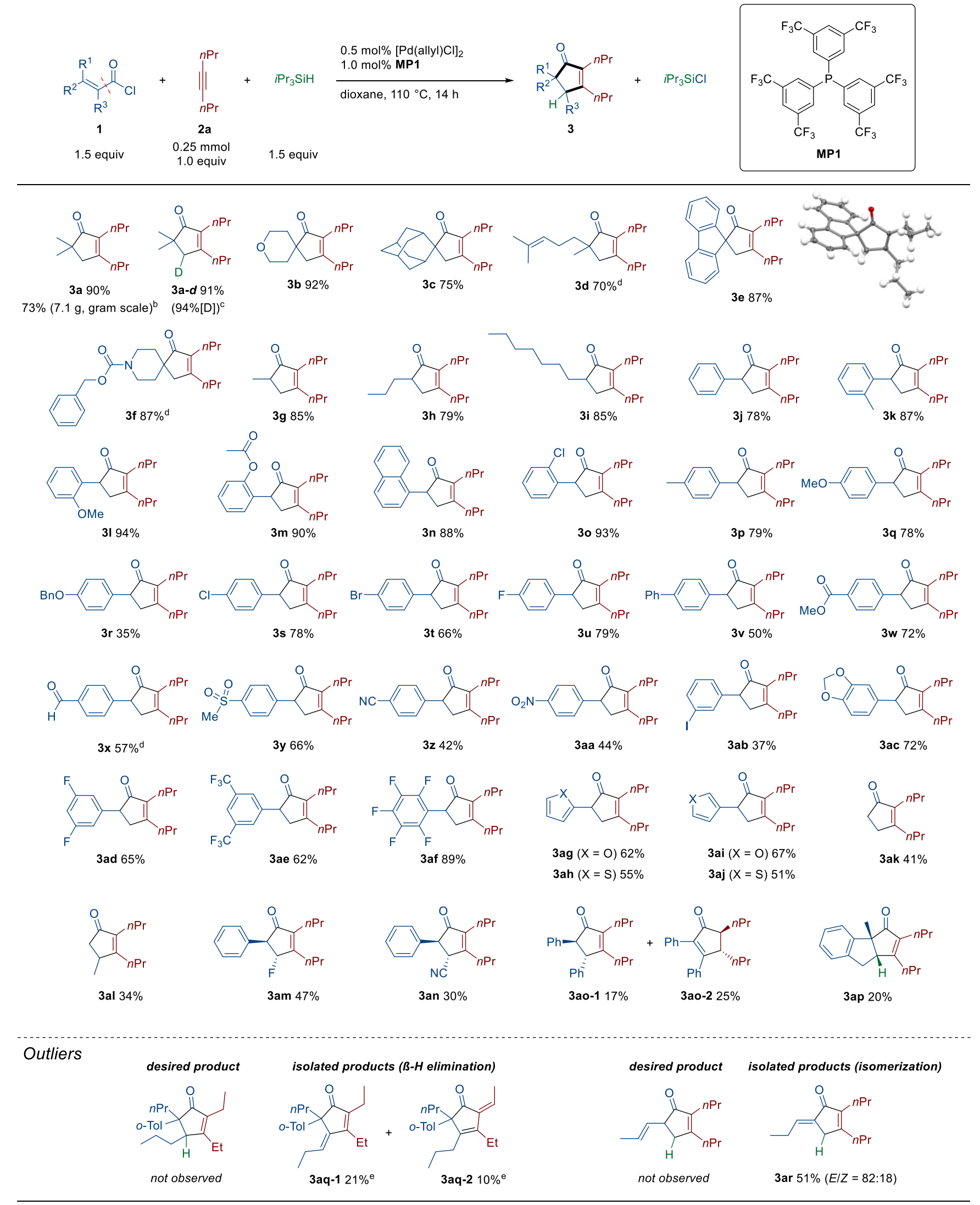

${ }^{a}$ All yields are isolated as a single regioisomer unless stated otherwise. ${ }^{b}$ Open system, $50 \mathrm{mmol} \mathrm{scale}$. ${ }^{\mathrm{c}} \mathrm{Pr}_{3} \mathrm{SiD}(1.5$ equiv. $){ }^{\mathrm{d}} \mathrm{The}$ corresponding carboxylic acid (1.5 equiv.) and Ghosez's reagent (1.5 equiv.) instead of 1. 3 -hexyne (1.5 equiv.) instead of 2 a. For details, see SI.

Table 3. Scope with respect to internal alkynes ${ }^{a}$ 


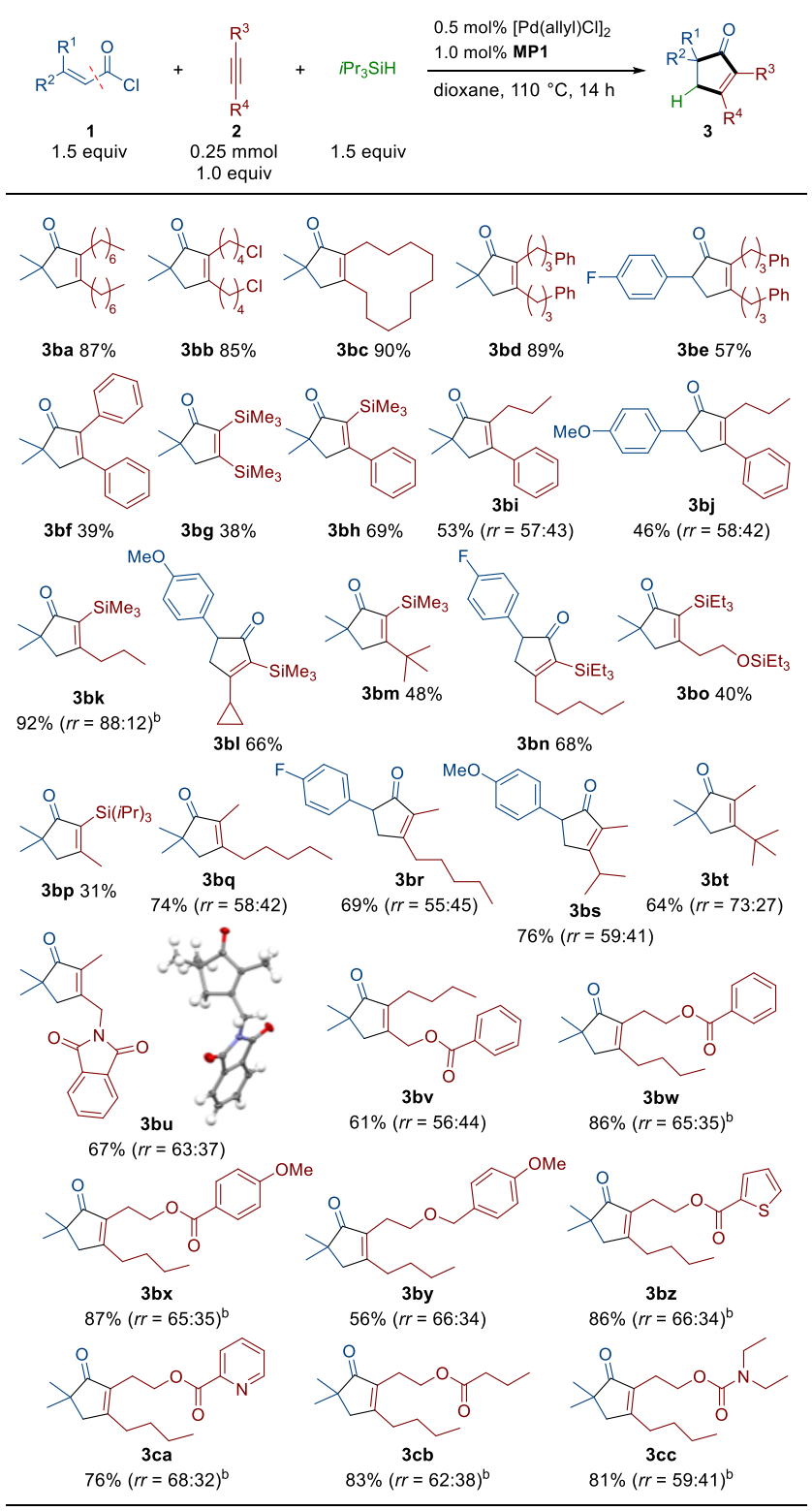

${ }^{a}$ All yields are isolated as a single regioisomer unless otherwise stated. bIsolated as an inseparable mixture of regioisomers. For details, see SI.

systems were examined. 3-methyl- $1 H$-indene-2-carbonyl chloride, a ring-fused tetrasubstituted enoyl chloride affords the desired tricyclic compound (3ap) as a single diastereomer. When an acyclic tetrasubstituted enoyl chloride was subjected to the reaction conditions, two isomers (3aq-1 and 3aq-2) were produced, which may result from an acyl Mizoroki-Heck-type coupling followed by $\beta-\mathrm{H}$ elimination. ${ }^{2,20}$ It is possible that the steric bulk installed into the substrate prevents the desired product formation. From these studies, it appears that the reaction is sensitive to steric encumbrance at the $\alpha$ position of the acid chloride, presumably resulting from a less favorable carbometallation step of the alkenyl-Pd species into an alkyne due to increased steric bulk. Gratifyingly, the developed conditions were easily translated to multigram scale $(50 \mathrm{mmol})$ experiments, affording compound $3 \mathrm{a}$ in $73 \%$ yield $(7.1 \mathrm{~g})$.

A set of functionalized dialkyl alkynes reacted efficiently (Table 3). Additionally, a cyclic alkyne (3bc), diarylalkyne (3bf) and even a bis-trimethylsilyl alkyne ( $\mathbf{3 b g}$ ) all undergo the reaction in moderate to excellent yields. The reaction is compatible with alkyl-silyl (3bk-3bp) and aryl-silyl (3bh) alkynes, furnishing the corresponding products with excellent regioselectivity, offering a versatile functional handle for further decoration. ${ }^{30}$ It is worth noting that the extremely bulky triisopropylsilyl group ( $\mathbf{3 b} \mathbf{b}$ ) is also tolerated, without any erosion of regioselectivity. Additionally, several functionalities appended to the alkyne, such as alkyl chlorides (3bb), phthalimides (3bu), esters (3bv-3bx, $\mathbf{3} \mathbf{c b}$ ), carbamates (3cc), silyl ethers (3bo) and $S$ - and $N$-heteroaryl groups ( $\mathbf{3 b z}$ and $\mathbf{3} \mathbf{c a}$ ), were well tolerated. While negligible regioselectivity was observed for unsymmetrical aryl-alkyl (3bi, $\mathbf{3 b j}$ ) and alkyl-alkyl (3bq-3bv) alkynes, a modest improvement resulted from using a sterically differentiated unsymmetrical alkyne (3bt), with a preference for alkenyl insertion at the more sterically encumbered position, presumably to minimize steric repulsion during carbopalladation. However, a polar functionality installed at the $\beta$-position relative to the alkyne (3bw-3cc) leads to a slight preference for the opposite regioisomer, resulting in the directing group tether being situated at the 2-position of the corresponding cyclopentenone. Terminal alkynes did not provide the corresponding cyclopentenones, while conversion of the alkyne was observed suggesting other processes such as polyaddition are operative.

Four interesting products can in theory be obtained from the combination of our three starting materials and reagents. While $\mathbf{3 a}$ was the only observed product under the standard reaction conditions (see SI), it was found that, in the absence of a phosphine ligand, its regioisomer $(\mathbf{4 a})$, from a formal reductive $[3+2]$ cyclization, is formed, ${ }^{19,20 c}$ demonstrating ligand-controlled regioselectivity (Scheme 2a). ${ }^{31}$ The modulated reactivity of the system in the presence of a phosphine ligand is thus crucial to allow the proposed fundamental steps of the reaction to occur in the right sequence. Further, $\mathrm{Rh}(\mathrm{I})$, a popular catalyst for carbonylation chemistry, showed a distinct reactivity with $\alpha, \beta$-unsaturated acid chlorides, forming the hydroacylated product (5a) stereoselectively with an unoptimized yield of $59 \%{ }^{32}$ Thus, this metal-based chemodivergence can also lead to useful substrates for subsequent Nazarov-type reactions (Scheme 2a). ${ }^{8,9}$

During our studies, we found that subjecting phenylacetyl chloride to the reaction conditions resulted in reductive decarbonylation of the acid chloride, resulting in the formation of toluene (see SI).,33 We thus hypothesized that this compound could serve as a novel CO-surrogate in carbonylation reactions. ${ }^{15,22 g, 24}$ Intriguingly, a fourcomponent coupling was achieved when phenylacetyl chloride, acting as a CO-equivalent, was coupled with a vinyl iodide, acting as an alkene partner, to deliver the cyclopentenone product (3as) in moderate yield with excellent regioselectivity (Scheme $2 \mathrm{~b}$ ). ${ }^{20,34}$ Other vinyl (pseudo)halides required harsher reaction conditions and/or an exogenous chloride source to increase their conversion (3at and 3au). This unique reactivity demonstrates that a variety of vinyl (pseudo)halides can also be used in a four-component coupling to deliver the desired cyclopentenone products.

Interestingly, the use of an aryl stannane as a carbon nucleophile, in place of the hydride nucleophile led to the pentasubstituted product (3av) in 58\% yield. ${ }^{35}$ This is another class of compound which is not directly accessible through PK-type reactions (Scheme 2c). 
In conclusion, we have developed a CO-free intermolecular synthesis of cyclopentenones using alkynes, $\alpha, \beta$-unsaturated acid chlorides and a hydrosilane taking advantage of a molecular shuffling process. Further, we showcased the benefit of using a molecular shuffling strategy by extending the reactivity to divergent processes, building on the potential of this concept as a novel strategy to develop a vast array of $\mathrm{CO}$-free carbonylation reactions.

\section{Scheme 2. Synthetic applications ${ }^{\mathrm{a}}$}

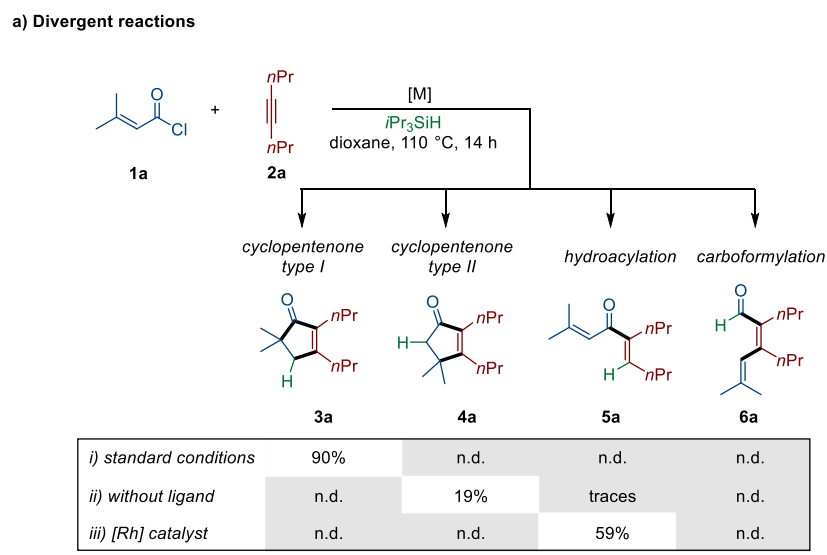

b) Phenylacetyl chloride as a new CO-equivalent in a 4-component coupling

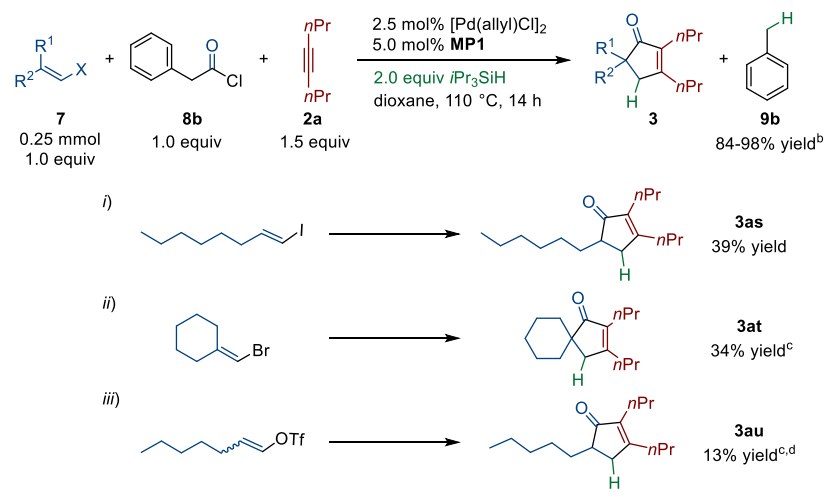

c) C-Nucleophile to generate pentasubstituted cyclopentenones
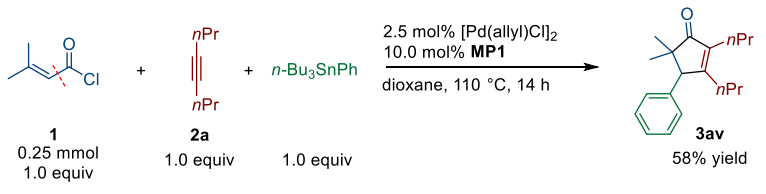

${ }^{\mathrm{a}}$ All yields are isolated as a single isomer. ${ }^{\mathrm{b}} \mathrm{GC}$ yields. ${ }^{\mathrm{c}} 130{ }^{\circ} \mathrm{C}$. ${ }^{\mathrm{d}} \mathrm{LiCl}(2$ equiv.) was added. For details, see SI.

\section{ASSOCIATED CONTENT}

Supporting Information. This material is available free of charge via the Internet at http://pubs.acs.org.

Experimental details for all reactions and analytic details for all products (PDF)

Crystallographic data (CIF, CCDC 2035178, compound 3e) Crystallographic data (CIF, CCDC 2045251, compound 31) Crystallographic data (CIF, CCDC 2045252, compound 3v) Crystallographic data (CIF, CCDC 2035179, compound 3bu-1, major)

\section{AUTHOR INFORMATION}

\section{Corresponding Author}

bill.morandi@org.chem.ethz.ch

\section{Funding Sources}

The financial support by the European Research Council under the European Union's Horizon 2020 research and innovation program (Shuttle Cat, Project ID: 757608), the ETH Zürich and LG Chem (fellowship to Y.H.L.) are gratefully acknowledged.

Notes

The authors declare no competing financial interest.

\section{ACKNOWLEDGMENT}

We thank the NMR, MS (MoBiAS) and X-ray (SMoCC) service departments at ETH Zürich for technical assistance. We are also grateful to Prof. E. M. Carreira for sharing chiral GC and M. Isomura and V. Gerken for technical help.

\section{REFERENCES}

(1) (a) Tietze, L. F. Domino Reactions in Organic Synthesis. Chem. Rev. 1996, 96, 115-136. (b) Nicolaou, K. C.; Edmonds, D. J.; Bulger, P. G. Cascade Reactions in Total Synthesis. Angew. Chem., Int. Ed. 2006, 45, 7134-7186. (c) Ardkhean, R.; Caputo, D. F. J.; Morrow, S. M.; Shi, H.; Xiong, Y.; Anderson, E. A. Cascade Polycyclizations in Natural Product Synthesis. Chem. Soc. Rev. 2016, 45, 1557-1569.

(2) (a) Negishi, E.; Copéret, C.; Ma, S.; Liou, S.-Y.; Liu, F. Cyclic Carbopalladation. A Versatile Synthetic Methodology for the Construction of Cyclic Organic Compounds. Chem. Rev. 1996, 96, 365-393. (b) Trost, B. M.; Krische, M. J. Transition Metal Catalyzed Cycloisomerizations. Synlett 1998, 1-16. (c) Büschleb, M.; Dorich, S.; Hanessian, S.; Tao, D.; Schenthal, K. B.; Overman, L. E. Synthetic Strategies toward Natural Products Containing Contiguous Stereogenic Quaternary Carbon Atoms. Angew. Chem., Int. Ed. 2016, 55, 4156-4186. (d) Grigg, R.; Sridharan V. Palladium Catalysed Cascade Cyclisation-Anion Capture, Relay Switches and Molecular Queues. J. Organomet. Chem. 1999, 576, 65-87. (e) Bai, Y.; Davis, D. C.; Dai, M. Natural Product Synthesis via Palladium-Catalyzed Carbonylation.J. Org. Chem. 2017, 82, 2319-2328. (f) Marchese, A. D.; Larin, E. M.; Mirabi, B.; Lautens, M. Metal-Catalyzed Approaches toward the Oxindole Core. Acc. Chem. Res. 2020, 53, 1605-1619.

(3) Touré, B. B.; Hall, D. G. Multicomponent Reactions in the Total Synthesis of Natural Products. In Multicomponent Reactions; Zhu, J., Bienaymé H., Eds.; Wiley-VCH: Weinheim, 2005; pp 342-397.

(4) Lee, Y. H.; Denton, E. H.; Morandi, B. Palladium-Catalyzed Carboformylation Enabled by a Molecular Shuffling Process. ChemRxiv. Preprint. https://doi.org/10.26434/chemrxiv.12624869.v1.

(5) (a) Simeonov, S. P.; Nunes, J. P. M.; Guerra, K.; Kurteva, V. B.; Afonso, C. A. M. Synthesis of Chiral Cyclopentenones. Chem. Rev. 2016, 116, 5744-5893. (b) Aitken,D.J.; Eijsberg, H.; Frongia, A.; Ollivier,J.; Piras, P. P. Recent Progress in the Synthetic Assembly of 2-Cyclopentenones. Synthesis 2014, 46, 1-24.

(6) (a) Chuang, K. V.; Xu, C.; Reisman, S. E. A 15-Step Synthesis of (+)-Ryanodol. Science 2016, 353, 912-915. (b) Dibrell, S. E.; Maser, M. R.; Reisman, S. E. SeO $\mathrm{S}_{2}$-Mediated Oxidative Transposition of Pauson-Khand Products. J. Am. Chem. Soc. 2020, 142, 6483-6487. (c) Liang, X.-T.; Chen, J.-H.; Yang, Z. Asymmetric Total Synthesis of (-)-Spirochensilide A. J. Am. Chem. Soc. 2020, 142, 8116-8121. (d) Qu, Y.; Wang, Z.; Zhang, Z.; Zhang, W.; Huang, J.; Yang, Z. Asymmetric Total Synthesis of (+)-Waihoensene. J. Am. Chem. Soc. 2020, 142, 6511-6515. (e) Hu, X.; Musacchio, A. J.; Shen, 
X.; Tao, Y.; Maimone, T. J. Allylative Approaches to the Synthesis of Complex Guaianolide Sesquiterpenes from Apiaceae and Asteraceae. J. Am. Chem. Soc. 2019, 141, 14904-14915. (f) Jørgensen, L.; McKerrall, S. J.; Kuttruff, C. A.; Ungeheuer, F.; Felding, J.; Baran, P. S. 14-Step Synthesis of (+)-Ingenol from (+)-3-Carene. Science 2013, 341, 878-882. (g) Hugelshofer, C. L.; Palani, V.; Sarpong, R. Calyciphylline B-Type Alkaloids: Total Syntheses of (-)-Daphlongamine $\mathrm{H}$ and (-)-Isodaphlongamine H. J. Am. Chem. Soc. 2019, 141, 8431-8435. (h) Nakayama, A.; Kogure, N.; Kitajima, M.; Takayama, H.Asymmetric Total Synthesis of a Pentacyclic Lycopodium Alkaloid: Huperzine-Q. Angew. Chem., Int. Ed. 2011, 50, 8025-8028. (i) Zweig, J. E.; Kim,D. E.; Newhouse, T. R. Methods Utilizing First-Row Transition Metals in Natural Product Total Synthesis. Chem. Rev. 2017, 117, 11680-11752. (j) Ma, K.; Martin, B. S.; Yin, X.; Dai, M. Natural Product Syntheses via Carbonylative Cyclizations. Nat. Prod. Rep., 2019, 36, 174 219.

(7) (a) Khand, I. U.; Knox, G. R.; Pauson, P. L.; Watts, W. E. A Cobalt Induced Cleavage Reaction and a New Series of Arenecobalt Carbonyl Complexes. J. Chem. Soc. D 1971, 36a. (b) Werner, H. Peter Ludwig Pauson (1925-2013). Angew. Chem. 2014, 126, 3375.

(8) (a) Jeong, N. The Pauson-Khand Reaction. In Comprehensive Organic Synthesis II, 2nd ed.; Knochel, P., Molander, G. A., Eds.; Elsevier: Amsterdam, 2014; Vol. 5, pp 1106-1178. (b) Schore, N. E. Pauson-Khand Cycloaddition Reaction for Synthesis of Cyclopentenones. Org. React. 1991, 40, 1-90. (c) Lindsay, D. M.; Kerr, W. J. Recent Advances in the PausonKhand Reaction. In Cobalt Catalysis in Organic Synthesis: Methods and Reactions, 1st ed.; Hapke, M., Hilt, G., Eds.; Wiley-VCH: Weinheim, 2020; pp 259-285. (d) Boñaga, L. V. R.; Krafft, M. E. When the Pauson-Khand and Pauson-Khand Type Reactions Go Awry: a Plethora of Unexpected Results. Tetrahedron 2004, 60, 9795-9833. (e) Zhang, S.; Neumann, H.; Beller, M. Synthesis of $\alpha, \beta$-Unsaturated Carbonyl Compounds by Carbonylation Reactions. Chem. Soc. Rev. 2020, 49, 3187-3210.

(9) (a) A. J. Frontier, J. J. Hernandez, New Twists in Nazarov Cyclization Chemistry. Acc. Chem. Res. 2020, 53, 1822-1832. (b) Tius, M. A. Some New Nazarov Chemistry. Eur. J. Org. Chem. 2005, 2193-2206. (c) Habermas, K. L.; Denmark, S. E.; Jones, T. K. The Nazarov Cyclization. Org. React. 1994, 45, 1-158.

(10) (a) Yadykov, A. V.; Shirinian, V.Z. Recent Advances in the Interrupted Nazarov Reaction. Adv. Synth. Catal. 2020, 362, 702-723. (b) Grant, T. N.; Riedera, C.J.; West, F. G. Interrupting the Nazarov Reaction: Domino and Cascade Processes Utilizing Cyclopentenyl Cations. Chem. Commun. 2009, 5676-5688.

(11) (a) Gibson, S. E.; Mainolfi, N. The Intermolecular PausonKhand Reaction. Angew. Chem., Int. Ed. 2005, 44, 3022-3037. (b) Laschat, S.; Becheanu, A.; Bell, T.; Baro, A. Regioselectivity, Stereoselectivity and Catalysis in Intermolecular Pauson-Khand Reactions: Teaching an Old Dog New Tricks. Synlett 2005, 2547-2570. (c) Su, S.; Rodriguez, R. A.; Baran, P. S. Scalable, Stereocontrolled Total Syntheses of ( \pm )-Axinellamines A and B. J. Am. Chem. Soc. 2011, 133, 13922-13925. (d) Chan, J.; Jamison, T. F. Enantioselective Synthesis of (-)-Terpestacin and Structural Revision of Siccanol Using Catalytic Stereoselective Fragment Couplings and Macrocyclizations. J. Am. Chem. Soc. 2004, 126, 10682-10691.

(12) (a) de Bruin, T. J. M.; Milet, A.; Greene, A. E.; Gimbert, Y. Insight into the Reactivity of Olefins in the Pauson-Khand Reaction. J. Org. Chem. 2004, 69, 1075-1080. (b) Jeong, N.; Chung, Y. K.; Lee, B. Y.; Lee, S. H.; Yoo, S.-E. A Dramatic Acceleration of the Pauson-Khand Reaction by Trimethylamine N-Oxide. Synlett 1991, 204-206. (c) Chung, Y. K.; Lee, B. Y.; Jeong, N.; Hudecek, M.; Pauson, P. L. Promoters for the (Alkyne) Hexacarbonyldicobalt-based Cyclopentenone Synthesis. Organometallics 1993, 12, 220-223. (d) Sugihara, T.; Yamada, M.; Yamaguchi, M.; Nishizawa, M. The Intra- and Intermolecular Pauson-Khand Reaction Promoted by Alkyl Methyl Sulfides. Synlett 1999, 771-773. (e) Marchueta, I.; Verdaguer, X.; Moyano, A.; Pericàs, M. A.; Riera, A. Intermolecular Pauson-Khand Reactions of Cyclopropene: A General Synthesis of Cyclopentanones. Org. Lett. 2001, 3, 3193-3196.
(13) (a) Krafft, M. E. Regiocontrol in the Intermolecular Cobalt-Catalyzed Olefin-Acetylene Cycloaddition. J. Am. Chem. Soc. 1988, 110, 968970. (b) Krafft, M. E.; Juliano, C. A.; Scott, I. L.; Wright, C.; McEachin, M. D. The Directed Pauson-Khand Reaction. J. Am. Chem. Soc. 1991, 113, 1693-1703. (c) Itami, K.; Mitsudo, K.; Yoshida, J. A Pyridylsilyl Group Expands the Scope of Catalytic Intermolecular Pauson-Khand Reactions. Angew. Chem., Int. Ed. 2002, 41, 3481-3484. (d) Itami, K.; Mitsudo, K.; Fujita, K.; Ohashi, Y.; Yoshida, J. Catalytic Intermolecular Pauson-Khand-Type Reaction: Strong Directing Effect of Pyridylsilyl and Pyrimidylsilyl Groups and Isolation of Ru Complexes Relevant to Catalytic Reaction. J. Am. Chem. Soc. 2004, 126, 11058-11066. (e) Rodríguez Rivero, M.; de la Rosa, J. C.; Carretero,J. C. Asymmetric Intermolecular Pauson-Khand Reactions of Unstrained Olefins: The (o-Dimethylamino)phenylsulfinyl Group as an Efficient Chiral Auxiliary. J. Am. Chem. Soc. 2003, 125, 14992-14993.

(14) Gallagher, A. G.; Tian, H.; Torres-Herrera, O. A.; Yin, S.; Xie, A.; Lange, D. M.; Wilson, J. K.; Mueller, L. G.; Gau, M. R.; Carroll, P. J.; Martinez-Solorio, D. Access to Highly Functionalized Cyclopentenones via Diastereoselective Pauson-Khand Reaction of Siloxy-Tethered 1,7-Enynes. Org. Lett. 2019, 21, 8646-8651.

(15) (a) Morimoto, T.; Fuji, K.; Tsutsumi, K.; Kakiuchi, K. COTransfer Carbonylation Reactions. A Catalytic Pauson-Khand-Type Reaction of Enynes with Aldehydes as a Source of Carbon Monoxide. J. Am. Chem. Soc. 2002, 124, 3806-3807. (b) Shibata, T.; Toshida N.; Takagi, K. Catalytic Pauson-Khand-Type Reaction Using Aldehydes as a CO Source. Org. Lett. 2002, 4, 1619-1621. (c) Fuji, K.; Morimoto, T.; Tsutsumi, K.; Kakiuchi, K. Aqueous Catalytic Pauson-Khand-Type Reactions of Enynes with Formaldehyde: Transfer Carbonylation Involving an Aqueous Decarbonylation and a Micellar Carbonylation. Angew. Chem., Int. Ed. 2003, 42, 2409 2411. (d) Park, K. H.; Son, S. U.; Chung, Y. K. Immobilized Heterobimetallic Ru/Co Nanoparticle-Catalyzed Pauson-Khand-Type Reactions in the Presence of Pyridylmethyl Formate. Chem. Commun. 2003, 1898-1899. (e) Morimoto, T.; Kakiuchi, K. Evolution of Carbonylation Catalysis: No Need for Carbon Monoxide. Angew. Chem., Int.Ed. 2004, 43, 5580-5588. (f) Park, J. H.; Cho, Y.; Chung, Y. K. Rhodium-Catalyzed Pauson-Khand-Type Reaction Using Alcohol as a Source of Carbon Monoxide. Angew. Chem., Int. Ed. 2010, 49, 5138-5141.

(16) (a) Jeong, N.; Hwang, S. H.; Lee, Y. Catalytic Version of the Intramolecular Pauson-Khand Reaction. J. Am. Chem. Soc. 1994, 116, 3159 3160. (b) Wang, Y.; Xu, L.; Yu, R.; Chen, J.; Yang, Z. CoBra-TMTU-Zinc Catalysed-Pauson-Khand Reaction. Chem. Commun. 2012, 48, 8183-8185. (c) Kim, S.-W.; Son, S. U.; Lee, S. I.; Hyeon, T.; Chung, Y. K. Cobalt on Mesoporous Silica: The First Heterogeneous Pauson-Khand Catalyst. J. Am. Chem. Soc. 2000, 122, 1550-1551. (d) Muller, J.-L.; Klankermayer, J.; Leitner, W. Poly(ethylene glycol) Stabilized Co Nanoparticles as Highly Active and Selective Catalysts for the Pauson-Khand Reaction. Chem. Commun. 2007, 1939-1941. (e) Shambayani, S.; Crowe, W. E.; Schreiber, S. L. N-Oxide Promoted Pauson-Khand Cyclizations at Room Temperature. Tetrahedron Lett. 1990, 31, 5289-5292.

(17) (a) Buchwald, S. L.; Hicks, F. A. Pauson- Khand Type Reactions. In Comprehensive Asymmetric Catalysis I-III; Jacobsen, E. N., Pfaltz, A., Yamamoto, H., Eds.; Springer-Verlag: Berlin, 1999; Vol. 2, pp 491-510. (b) Jeong, N.; Lee, S.; Sung, B. K. Rhodium(I)-Catalyzed Intramolecular Pauson-Khand Reaction. Organometallics 1998, 17, 3642-3644. (c) Koga, Y.; Kobayashi, T.; Narasaka, K. Rhodium-Catalyzed Intramolecular Pauson-Khand Reaction. Chem. Lett. 1998, 27, 249-250. (d) Wender, P. A.; Deschamps, N. M.; Williams, T. J. Intermolecular Dienyl Pauson-Khand Reaction, Angew. Chem., Int. Ed. 2004, 43, 3076-3079. (e) Shibata, T.; Takagi, K. Iridium-Chiral Diphosphine Complex Catalyzed Highly Enantioselective Pauson-Khand-Type Reaction. J. Am. Chem. Soc. 2000, 122, $9852-$ 9853. (f) Grigg, R.; Zhang, L.; Collard, S.; Keep, A. Palladium Catalysed $[2+2+1]$ Intramolecular Cycloaddition for the Preparation of Bicyclo[3.3.0] octa-1.5-dien-3-ones from 1,6-Diynes. Chem. Commun. 2003, 1902-1903. (g) Huang, Z.; Huang, J.; Qu, Y.; Zhang, W.; Gong, J.; Yang, Z. Total Syntheses of Crinipellins Enabled by Cobalt-Mediated and Palladium- 
Catalyzed Intramolecular Pauson-Khand Reactions. Angew. Chem., Int. Ed. 2018, 57, 8744-8748.

(18) Park, K. H.; Jung, I. G.; Chung, Y. K. A Pauson-Khand-Type Reaction between Alkynes and Olefinic Aldehydes Catalyzed by Rhodium/Cobalt Heterobimetallic Nanoparticles: An Olefinic Aldehyde as an Olefin and CO Source. Org. Lett. 2004, 6, 1183-1186.

(19) (a) Ohashi, M.; Taniguchi, T.; Ogoshi, S. Nickel-Catalyzed Formation of Cyclopentenone Derivatives via the Unique Cycloaddition of $\alpha, \beta-$ Unsaturated Phenyl Esters with Alkynes. J. Am. Chem. Soc. 2011, 133, 14900-14903. (b) Jenkins, A. D.; Herath, A.; Song, M.; Montgomery,J. Synthesis of Cyclopentenols and Cyclopentenones via Nickel-Catalyzed Reductive Cycloaddition. J. Am. Chem. Soc. 2011, 133, 14460-14466. (c) Ahlin, J. S. E.; Donets, P. A.; Cramer, N. Nickel(0)-Catalyzed Enantioselective Annulations of Alkynes and Arylenoates Enabled by a Chiral NHC Ligand: Efficient Access to Cyclopentenones. Angew. Chem., Int. Ed. 2014, 53, 1322913233. (d) Jenkins, A. D.; Robo, M. T.; Zimmerman, P. M.; Montgomery, J. Nickel-Catalyzed Three-Component Cycloadditions of Enoates, Alkynes, and Aldehydes. J. Org. Chem. 2020, 85, 2956-2965. (e) Barluenga, J.; Barrio, P.; Riesgo, L.; López, L. A.; Tomás, M. A General and Regioselective Synthesis of Cyclopentenone Derivatives through Nickel(0)-Mediated [3+2] Cyclization of Alkenyl Fischer Carbene Complexes and Internal Alkynes. J. Am. Chem. Soc. 2007, 129, 46, 14422-14426. (f) Barluenga, J.; Álvarez-Fernández, A.; Suárez-Sobrino, Á. L.; Tomás, M. Regio- and Stereoselective Synthesis of Cyclopentenones: Intermolecular Pseudo-Pauson-Khand Cyclization. Angew. Chem., Int. Ed. 2012, 51, 183-186. (g) Rizzo, C. J.; Dunlap, N. K.; Smith III, A. B. A Convenient Preparation of 4 and 5-Substituted Cyclopentenones: a Short Synthesis of Methylenomycin B. J. Org. Chem. 1987, $52,5280-5283$.

(20) (a) Negishi, E.; Copéret,C.; Ma, S.; Mita,T.; Sugihara,T.; Tour, J. M. Palladium-Catalyzed Carbonylative Cyclization of 1-Iodo-2alkenylbenzenes. J. Am. Chem. Soc. 1996, 118, 5904-5918. (b) Negishi, E.; Ma, S.; Amanfu, J.; Copéret, C.; Miller, J. A.; Tour, J. M. Palladium-Catalyzed Cyclization of 1-Iodo-Substituted 1,4-, 1,5-, and 1,6-Dienes as Well as of 5-Iodo-1,5-dienes in the Presence of Carbon Monoxide. J. Am. Chem. Soc. 1996, 118, 5919-5931. (c) Copéret, C.; Sugihara, T.; Wu, G.; Shimoyama, I.; Negishi, E. Acylpalladation of Internal Alkynes and Palladium-Catalyzed Carbonylation of (Z)- $\beta$-Iodoenones and Related Derivatives Producing $\gamma$ Lactones and $\gamma$-Lactams. J. Am. Chem. Soc. 1995, 117, 3422-3431. (d) Sugihara, T.; Copéret, C.; Owczarczyk, Z.; Harring, L. S.; Negishi E. Deferred Carbonylative Esterification in the Pd-Catalyzed Cyclic CarbometalationCarbonylation Cascade. J. Am. Chem. Soc. 1994, 116, 7923-7924. (e) Gagnier, S. V.; Larock, R. C.Palladium-Catalyzed Carbonylative Cyclization of Unsaturated Aryl Iodides and Dienyl Triflates, Iodides, and Bromides to Indanones and 2-Cyclopentenones. J. Am. Chem. Soc. 2003, 125, 48044807.

(21) (a) Larock, R. C.; Oertle, K.; Potter, G. F. A Convenient Synthesis of Cyclopentanones via Rhodium(I)-Catalyzed Intramolecular Hydroacylation of Unsaturated Aldehydes. J. Am. Chem. Soc. 1980, 102, 190-197. (b) Tanaka, K.; Fu, G. C. A Versatile New Method for the Synthesis of Cyclopentenones via an Unusual Rhodium-Catalyzed Intramolecular Trans Hydroacylation of an Alkyne. J. Am. Chem. Soc. 2001, 123, 11492-11493.

(22) (a) Torres, G. M.; Liu, Y.; Arndtsen, B. A. A Dual Light-Driven Palladium Catalyst: Breaking the Barriers in Carbonylation Reactions. Science 2020, 368, 318-323. (b) Quesnel, J. S.; Arndtsen, B. A. A PalladiumCatalyzed Carbonylation Approach to Acid Chloride Synthesis.J. Am. Chem. Soc. 2013, 135, 16841-16844. (c) Fang, X.; Cacherat, B.; Morandi, B. COand HCl-Free Synthesis of Acid Chlorides from Unsaturated Hydrocarbons via Shuttle Catalysis. Nat. Chem. 2017, 9, 1105-1109. (d) Lee, Y. H.; Morandi, B. Metathesis-Active Ligands Enable a Catalytic Functional Group Metathesis between Aroyl Chlorides and Aryl Iodides. Nat. Chem. 2018, 10, 1016-1022. (e) De La Higuera Macias, M.; Arndtsen, B. A. Functional Group Transposition: a Palladium-Catalyzed Metathesis of Ar-X $\sigma$-Bonds and Acid Chloride Synthesis. J.Am. Chem. Soc. 2018, 140, 10140-10144. (f) Sakurai, Y.; Ogiwara, Y.; Sakai, N. Palladium-Catalyzed Annulation of Acyl Fluorides with Norbornene via Decarbonylation and CO Reinsertion. Chem.
Eur. J. 10.1002/chem.202001374.(g) Boehm, P.; Roediger, S.; Bismuto, A.; Morandi, B. Palladium-Catalyzed Chlorocarbonylation of Aryl (Pseudo)Halides Through In Situ Generation of Carbon Monoxide. Angew. Chem., Int. Ed. 2020, 59, 17887-17896.

(23) (a) Niemeyer, Z. L.; Milo1, A.; Hickey, D. P.; Sigman M. S. Parameterization of Phosphine Ligands Reveals Mechanistic Pathways and Predicts Reaction Outcomes. Nat. Chem. 2016, 8, 610-617. (b) Wu, K.; Doyle, A. G. Parameterization of Phosphine Ligands Demonstrates Enhancement of Nickel Catalysis via Remote Steric Effects. Nat. Chem. 2017, 9, 779-784.

(24) Friis, S. D.; Lindhardt, A. T.; Skrydstrup, T. The Development and Application of Two-Chamber Reactors and Carbon Monoxide Precursors for Safe Carbonylation Reactions. Acc. Chem. Res. 2016, 49, 594-605.

(25) (a) Smit, W. A.; Kireev, S. L.; Nefedov, O. M.; Tarasov, V. A. Methylenecyclopropane as an Alkene Component in the Khand-Pauson Reaction. Tetrahedron Lett. 1989, 30, 4021-4024. (b) Ishizaki, M.; Kasama, Y.; Zyo,M.; Niimi, Y.; Hoshino, O. A Facile Synthesis of Spiro-cyclopentenones from Various 1-Alkynes and Cyclic exo-Methylene Compounds by Intermolecular Pauson-Khand Reaction. Heterocycles 2001, 55, 1439-1442. (c) Corlay, H.; James, I. W.; Fouquet, E.; Schmidt, J.; Motherwell, W. B. Some Intermolecular Pauson-Khand Reactions of Functionalised Alkylidenecyclopropanes. Synlett 1996, 990-992. (d) Ishizaki, M.; Zyo, M.; Kasama, Y.; Niimi, Y.; Hoshino, O.; Nishitani, K.; Hara, H. Investigation of the Intermolecular Pauson-Khand Reaction of Various 1-Alkynes with Cyclic Exo-methylene Compounds. Heterocycles 2003, 60, 2259-2271.

(26) (a) Cleary, S. E.; Hensinger, M. J.; Brewer, M. Remote C-H Insertion of Vinyl Cations Leading to Cyclopentenones. Chem. Sci. 2017, 8, 6810-6814. (b) Krafft, M. E.; Cheung, Y. Y.; Abboud, K. A. Total Synthesis of ( \pm )-Asteriscanolide. J. Org. Chem. 2001, 66, 7443-7448. (c) Millham, A. B.; Kier, M. J.; Leon, R. M.; Karmakar, R.; Stempel, Z. D.; Micalizio, G. C. A Complementary Process to Pauson-Khand-Type Annulation Reactions for the Construction of Fully Substituted Cyclopentenones. Org. Lett. 2019, 21, 567-570.

(27) (a) Marchand-Brynaert, J.; Ghosez, L. Electrophilic Aminoalkenylation of Aromatics with $a$-Chloroenamines. J. Am. Chem. Soc. 1972, 94, 2869-2870. (b) Malapit, C. A.; Bour, J. R.; Brigham, C. E.; Sanford, M. S. Base-Free Nickel-Catalysed Decarbonylative Suzuki-Miyaura Coupling of Acid Fluorides. Nature 2018, 563, 100-104.

(28) (a) Cabré, A.; Khaizourane, H.; Garçon, M.; Verdaguer, X.; Riera, A. Total Synthesis of ( $R$ )-Sarkomycin Methyl Ester via Regioselective Intermolecular Pauson-Khand Reaction and Iridium-Catalyzed Asymmetric Isomerization. Org. Lett. 2018, 20, 3953-3957. (b) Jeong, N.; Hwang, S. H. Catalytic Intermolecular Pauson-Khand Reactions in Supercritical Ethylene. Angew. Chem., Int. Ed. 2000, 39, 636-638. (c) Rautenstrauch, V.; Mégard, P.; Conesa, J.; Küster, W. 2-Pentylcyclopent-2-en-1-one by Catalytic Pauson-Khand Reaction. Angew. Chem., Int. Ed. 1990, 29, 1413-1416.

(29) Román, R.; Mateu, N.; López, I.; Medio-Simon, M.; Fustero, S.; Barrio, P. Vinyl Fluorides: Competent Olefinic Counterparts in the Intramolecular Pauson-Khand Reaction. Org. Lett. 2019, 21, 2569-2573.

(30) Langkopf, E.; Schinzer, D. Uses of Silicon-Containing Compounds in the Synthesis of Natural Products. Chem. Rev. 1995, 95, 13751408.

(31) Andersson, C. M.; Hallberg, A. Palladium-Catalyzed Aroylation of Alkyl Vinyl Ethers. An Entry to Monoprotected 1-Aryl-1,3-Dicarbonyl Equivalents. J. Org. Chem. 1988, 53, 4257-4263.

(32) (a) Fujihara, T.; Tatsumi, K.; Terao, J.; Tsuji, Y. Palladium-Catalyzed Formal Hydroacylation of Allenes Employing Acid Chlorides and Hydrosilanes. Org. Lett. 2013, 15, 2286-2289. (b) Bandar, J. S.; Ascic, E.; Buchwald, S. L. Enantioselective CuH-Catalyzed Reductive Coupling of Aryl Alkenes and Activated Carboxylic Acids. J. Am. Chem. Soc. 2016, 138, 5821-5824. (c) Ueda, Y.; Iwai, T.; Sawamura, M. Nickel-Copper-Catalyzed Hydroacylation of Vinylarenes with Acyl Fluorides and Hydrosilanes. Chem. Eur. J. 2019, 25, 9410-9414. (d) Cheng, L.-J.; Mankad, N. P. Cu-Catalyzed Hydrocarbonylative C-C Coupling of Terminal Alkynes with Alkyl Iodides. J. Am. Chem. Soc. 2017, 139, 10200-10203. 
(33) (a) Ogiwara, Y.; Sakurai, Y.; Hattori, H.; Sakai, N. PalladiumCatalyzed Reductive Conversion of Acyl Fluorides via Ligand-Controlled Decarbonylation. Org. Lett. 2018, 20, 4204-4208. (b) Tsuji, J. PalladiumCatalyzed Decarbonylation of Acyl Halides and Aldehydes. In Handbook of Organopalladium Chemistry for Organic Synthesis; Negishi, E., Ed.; Wiley: New York, 2002; pp 2643-2653.
(34) Kerr, W. J.; McLaughlin, M.; Pauson, P. L.; Robertson, S. M. Vinyl Esters as Ethylene Equivalents in the Khand Annulation Reaction. Chem. Commun. 1999, 2171-2172.

(35) Milstein, D.; Stille, J. K. Mild, Selective, General Method of Ketone Synthesis from Acid Chlorides and Organotin Compounds Catalyzed by Palladium.J. Org. Chem. 1979, 44, 1613-1618. 
This document is the Accepted Manuscript version of a Published Work that appeared in final form in the Journal of the American Chemical Society, copyright (C) American Chemical Society after peer review and technical editing by the publisher. To access the final edited and published work see https://doi.org/10.1021/jacs.0c10832

\section{Table of Contents}

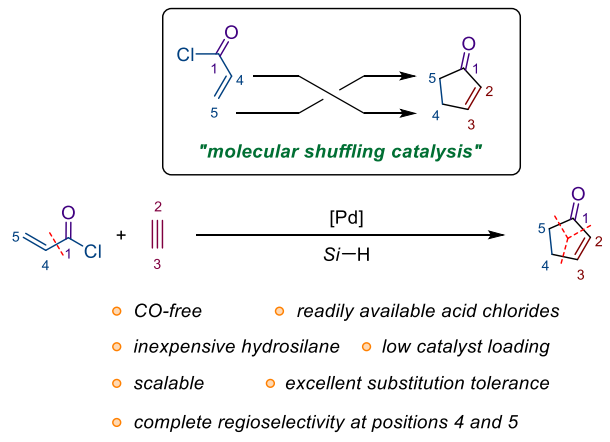

